\title{
The genetic variance but not the genetic covariance of life-history traits changes towards the north in a time-constrained insect
}

\author{
SZYMON SNIEGULA* DD, MARIA J. GOLAB*, SZYMON M. DROBNIAK† \& \\ FRANK JOHANSSON: \\ *Department of Ecosystem Conservation, Institute of Nature Conservation, Polish Academy of Sciences, Krakow, Poland \\ $\dagger$ Population Ecology Group, Institute of Environmental Sciences, Jagiellonian University, Krakow, Poland \\ $\$$ Department of Ecology and Genetics, Uppsala University, Uppsala, Sweden
}

\section{Keywords:}

genetic correlation;

G-matrix;

latitude:

life history;

seasonal time constraint.

\begin{abstract}
Seasonal time constraints are usually stronger at higher than lower latitudes and can exert strong selection on life-history traits and the correlations among these traits. To predict the response of life-history traits to environmental change along a latitudinal gradient, information must be obtained about genetic variance in traits and also genetic correlation between traits, that is the genetic variance-covariance matrix, G. Here, we estimated $\mathbf{G}$ for key life-history traits in an obligate univoltine damselfly that faces seasonal time constraints. We exposed populations to simulated native temperatures and photoperiods and common garden environmental conditions in a laboratory set-up. Despite differences in genetic variance in these traits between populations (lower variance at northern latitudes), there was no evidence for latitude-specific covariance of the life-history traits. At simulated native conditions, all populations showed strong genetic and phenotypic correlations between traits that shaped growth and development. The variancecovariance matrix changed considerably when populations were exposed to common garden conditions compared with the simulated natural conditions, showing the importance of environmentally induced changes in multivariate genetic structure. Our results highlight the importance of estimating variance-covariance matrixes in environments that mimic selection pressures and not only trait variances or mean trait values in common garden conditions for understanding the trait evolution across populations and environments.
\end{abstract}

\section{Introduction}

The additive genetic variance of a trait is a key parameter that determines evolutionary potential of the trait. However, organisms are not collections of isolated traits that evolve independently. Instead, they are composed of genetically, functionally and developmentally correlated traits that may adaptively (or maladaptively) covary across environments and populations (Stearns et al., 1991; Pigliucci \& Preston, 2004). Therefore, the level of genetic variance of individual traits and their genetic

Correspondence: Szymon Sniegula, Department of Ecosystem

Conservation, Institute of Nature Conservation, Polish Academy of Sciences, al. Mickiewicza 33, 31-120 Krakow, Poland.

Tel.: +48 1237035 22; fax: +48 1263224 32;

e-mail: szymon.sniegula@gmail.com covariance in specific environments will substantially affect evolution (Blows \& Hoffmann, 2005; Puentes et al., 2016). For example, when populations of the same species differ in their genetic variance and correlations among traits, these populations may differ in their response to environmental changes such as global warming (Etterson \& Shaw, 2001).

The genetic variance-covariance matrix, G, summarizes multivariate genetic structure of characters in focus. Hence, evaluation of size, shape and direction of G allows for robust predictions of evolutionary outcomes (Conner \& Hartl, 2004; Agrawal \& Stinchcombe, 2009). Ignorance of $\mathbf{G}$ when predicting evolutionary changes in traits might cause over- or underestimation of the evolutionary capacity of a trait (Prokop \& Drobniak, 2016). For example, although two leaf traits in 
Chamaecrista fasciculata showed substantial genetic variances, a strong negative genetic correlation between the traits explained considerably slower adaptive evolution of individual traits than would be expected if genetic covariance had been ignored (Etterson \& Shaw, 2001). By contrast, femur length in humans was not a character under direct selection along a latitudinal cline but continued to show change in evolutionary time because of its correlation with other limb characters (Savell et al., 2016).

The genetic variance-covariance matrix can vary considerably when organisms experience different environments (Stearns et al., 1991; Pigliucci, 2005; Doroszuk et al., 2008; Sikkink et al., 2015). Typically, changes in G require many generations when the change is driven by selection, migration, mutation and drift (Arnold et al., 2008). However, environmental effects on G may occur within a generation, which might happen because an environmental change releases genetic variation that was hidden in the past environment (McGuigan $\&$ Sgro, 2009). In support of strong environmental effects on G, Wood \& Brodie (2015) concluded in a review that variation in size, shape and direction of $\mathbf{G}$ can change as much or more between environments than between populations, indicating that short-term environmental effects on multivariate genetic structure can be as strong as the multigeneration effects across populations.

In addition to steady increases in annual ambient temperature (IPCC, 2013), unpredictable environmental extremes are predicted to become more frequent during global climate change (Bailey \& van de Pol, 2016). An increasing number of studies show that the current Gmatrix structure in a population can change considerably when the population experiences new realistic environmental conditions (Stoks et al., 2014; Bybee et al., 2016). Such studies are required because they reveal whether the environmental change imposed is sufficiently strong to cause a substantial change in $\mathbf{G}$, upon which natural selection can work (Wood \& Brodie, 2015).

In temperate regions, high-latitude populations of ectothermic animals are exposed to greater seasonal time constraints than those of low-latitude populations, because the growth season becomes progressively shorter and colder towards the geographic poles. Latitudinal compensating mechanisms by which high-latitude organisms compensate for the brief growth season by showing faster growth and development than would be otherwise expected have been widely documented (Dmitriew, 2011; Sniegula et al., 2012a, 2017; Orizaola et al., 2014). These compensating mechanisms could be caused by genetic change or phenotypic plasticity, with phenotypic plasticity referring to phenotypic changes of a given genotype in response to the experienced condition. Such adaptive latitudinal differentiation in life-history traits describes past evolutionary outcomes of those populations. Nevertheless, the level of evolutionary potential and genetic constraints in terms of a multivariate approach of traits has rarely been evaluated across latitudes (Kause et al., 2001; Colautti \& Barrett, 2011; Shama et al., 2011). With such information, we can understand the constraints and evolution of compensatory growth and development of organisms at high latitudes.

In this study, we examined latitudinal differentiation in the genetic variance-covariance of life-history traits using the damselfly Lestes sponsa (Hansemann). This damselfly has a wide latitudinal distribution (Boudot $\delta$ Kalkman, 2016) and therefore faces strong time constraints at high latitudes (Sniegula et al., 2016c). These constraints are magnified because this damselfly is obligate univoltine (one generation per season; Jödicke, 1996); thus, when conditions deteriorate, premature development cannot be prolonged by an extra season. As a result, directional or stabilizing selection on lifehistory traits should be stronger in northern populations because less time is available for growth and development. Thus, because of strong time constraints on life-history traits, genetic variation should be reduced in the north, and the same might hold for the genetic covariance. Therefore, we would expect that the G-matrix characteristics change along a latitudinal gradient in organisms that have an obligate 1-year life cycle. However, despite strong empirical support that fitness-related traits are common targets of selection and shape important ecological interactions (Dmitriew, 2011; Sniegula et al., 2016b), we lack a good understanding of the changes in the genetic variance-covariance matrix $(\mathbf{G})$ along a latitudinal gradient and in the structure of $\mathbf{G}$ when latitudinal populations are exposed to a new environment.

Because egg development time, larval development time and larval growth are important life-history traits that affect, for example, mating success and survival (De Block \& Stoks, 2005; O'Connor et al., 2014), but see Potter et al. (2011), the correlations of these traits along a latitudinal gradient are of interest. We estimated and compared pairwise variance-covariance structures for egg and larval development time and larval growth rate of $L$. sponsa originating from replicated high-, central- and low-latitude populations, separated by a total distance of $2730 \mathrm{~km}$. Variation in egg development time causes variation in hatching date, and hatching date determines the time period available for growth, development and reproduction (Gotthard, 2001). Similarly, variation in larval development and growth rate causes variation in time to and size at emergence, which should be optimized along a latitudinal gradient (Dmitriew, 2011). We asked the following questions: (Q1) What is the genetic variance and covariance structure of the three traits along a latitudinal gradient, and (Q2) how does the genetic variance and covariance structure of the three traits change 
when larvae are exposed to a new environment? To answer these questions, we raised full sibs under laboratory conditions. Q1 was answered by raising larvae in their native temperature and light regime conditions at their origin of collection (latitude). By simulating the seasonal change in these two environmental factors at the origin of the larval population, we simulated the time constraints the larvae were exposed to under natural conditions. Q2 was answered by raising larvae in a common garden environment using a constant temperature and light regime and therefore exposing larvae to a novel environment to which they had never been exposed.

\section{Materials and methods}

\section{Study species}

Lestes sponsa is a widespread and common species in Europe, with a broad north-south distribution (Boudot \& Kalkman, 2016; Fig. 1). This damselfly is an obligate univoltine species. The eggs, which are laid in summer, are the overwintering stage. Larvae hatch during the subsequent spring, and individuals emerge and mature in summer (Jödicke, 1996). Previous studies on the life history of $L$. sponsa indicate latitudinal differentiation in mean trait values and differences in additive genetic variance of life-history traits across latitudes. High-latitude populations that are seasonally time- and thermally constrained evolve more synchronous egg

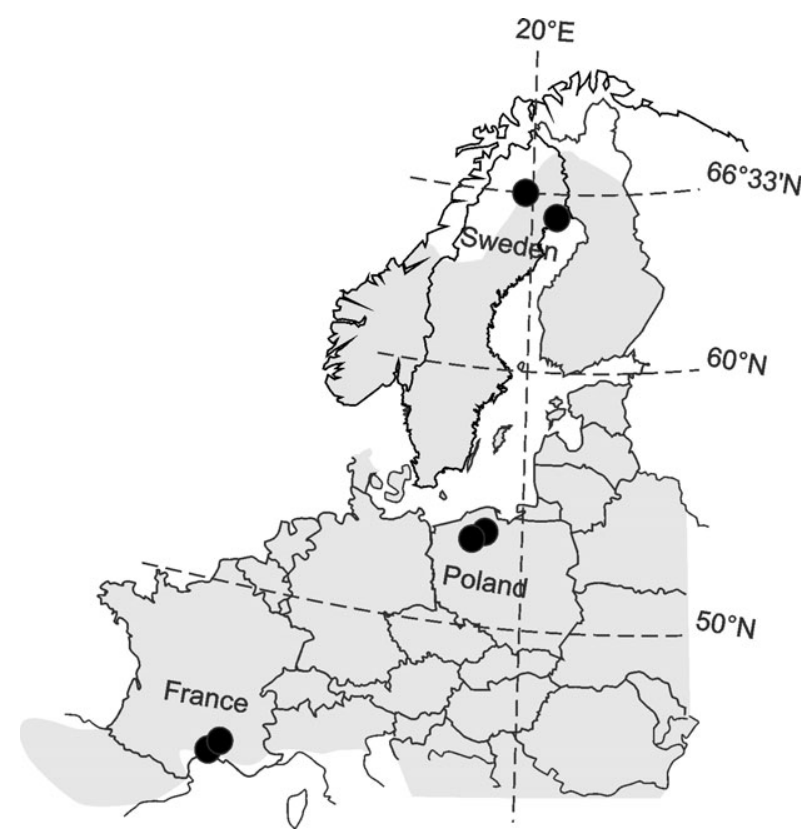

Fig. 1 Map showing the sampled populations (filled circles) and the European distribution of the damselfly Lestes sponsa (shaded part of the map: modified after Boudot \& Kalkman, 2016). development and hatching than central- and low-latitude populations, respectively (Sniegula et al., 2016c). High-latitude individuals also have faster and more synchronous larval growth and emergence than populations from central and low latitudes, respectively (Sniegula et al., 2016c). Similarly, lower additive genetic variance in egg development time and larval growth occurs in high-latitude populations than in central- and low-latitude populations, respectively (Sniegula et al., 2016a). Additional studies also show that day length (photoperiod) plays a key role in shaping these traits across different latitudes and is involved in compensatory growth at high latitudes (Sniegula \& Johansson, 2010; Sniegula et al., 2014). However, these previous studies have not quantified the genetic variance-covariance matrix $(\mathbf{G})$ between traits involved in compensatory mechanisms and whether the latitude of origin affects $\mathbf{G}$.

\section{Field sampling}

Two replicated populations in three latitudinal distant European regions were sampled (Fig. 1), which were high-, central- and low-latitude populations. Coordinates of sampled populations are given in Table S1. Note that the between-population distance in the replicated populations within all regions was small enough to allow extensive gene flow (Geenen et al., 2000), and previous studies on damselflies, including L. sponsa, show that differences in life histories within regions are smaller than those between regions (Shama et al., 2011; Sniegula et al., 2014). Field sampling of populations occurred in 2013 on the following dates: 29 June to 2 July at low latitude, 23 July to 28 July at central latitude and 6 August to 10 August at high latitude. For each population, we sampled mating females to receive full-sib families for the analysis, which was accomplished by catching males and females and then allowing them to mate in small field insectaries. Once mating occurred in the insectaries, the mated females were transferred to plastic jars with wet filter paper on a side for egg laying. We transported jars with females to a nearby indoor building at a temperature of $22^{\circ} \mathrm{C}$ with a natural photoperiod. Females were maintained in this building until eggs were deposited onto the wet filter paper, typically within $48 \mathrm{~h}$ after mating. From each population, females produced the following number of full-sib families: high latitude, 16 and 28; central latitude, 32 and 17; low latitude, 36 and 18. We supposed that offspring within each egg clutch contained full sibs, because the proportion of the female's offspring sired by the last male with whom she copulated is not less than 95\% (Corbet, 1999). Our design did not allow estimation of maternal effects, but Sniegula et al. (2016a) showed that maternal effects were low, for example maximum $12 \%$ in egg development time and $0.9 \%$ in larval growth rate in these populations. After 
egg deposition eggs were transported in darkness (in a cooler box at a temperature of $22{ }^{\circ} \mathrm{C}$ ) to a laboratory at the Institute of Nature Conservation PAS in Krakow, Poland. The transportation required between one (central-latitude populations) and three (high-latitude populations) days, and such transportation has no effect on L. sponsa development (Sniegula \& Johansson, 2010; Sniegula et al., 2014).

\section{Experiment 1: simulated conditions}

The purpose of this experiment was to estimate the genetic variance and covariance structure of the three traits along a latitudinal gradient under conditions that simulated those occurring at the origin of the sampled populations. For this purpose, individuals were raised from hatching until emergence in incubators for which we programmed conditions simulating natural temperatures and photoperiods (thermo-photoperiods) at the sampled latitudes. Three incubators were used at the Institute of Nature Conservation PAS in Krakow, Poland. To simulate seasonal changes in thermo-photoperiods, we changed the thermo-photoperiod once a week (every Friday), except in the winter simulation.

We obtained shallow water temperatures (optimal habitat for damselfly larvae, Corbet, 1999) during the growth season at each sampling latitude using the lake model FLake (Lake Model Flake, 2009). The photoperiod regimes that we applied included both morning and evening civil twilights at the latitude of each study site. We initiated weekly changes in latitude-specific thermo-photoperiods when eggs overwintered in the simulated winter conditions. Graphs showing temperatures and photoperiods used during experiment 1 are in Fig. S1.

After arrival in the laboratory, we placed egg clutches in plastic containers $(\mathrm{cm}$, height $5 \mathrm{~cm})$ with $250 \mathrm{~mL}$ of mixed dechlorinated tap and filtered pond water and placed these containers with eggs in the latitude-specific incubators with water temperature and light conditions resembling late summer at each sampled latitude. We used one container for each egg clutch. Temperatures for high-, central- and low-latitude incubators were $19.2{ }^{\circ} \mathrm{C}, 21{ }^{\circ} \mathrm{C}$ and $24.8^{\circ} \mathrm{C}$, respectively; photoperiods (L-D) for high-, central- and low-latitude incubators were 20:57-3:03, 17:38-6:22 and 16:31-7:29 h:min $\mathrm{L}-\mathrm{D}$, respectively. After 3 weeks of maintaining eggs in these thermo-photoperiods, we initiated winter conditions by first lowering temperature to $15{ }^{\circ} \mathrm{C}$ but maintaining the photoperiod. On the next day, we adjusted the temperature to $5{ }^{\circ} \mathrm{C}$ and set photoperiod to $\mathrm{L}-\mathrm{D}$ $0: 24 \mathrm{~h}$. All egg clutches were maintained in these winter conditions for 28 days.

We initiated spring conditions on dates when water temperature exceeded $12{ }^{\circ} \mathrm{C}$ (Lake Model Flake, 2009) at the origin of the latitudes sampled. We chose these temperatures and corresponding photoperiods because
L. sponsa begins to hatch when water temperature increases above $10{ }^{\circ} \mathrm{C}$ (Corbet, 1956). From these dates, we started simulating weekly changes of temperatures. As the larvae hatched, they were moved from the egg containers and introduced to plastic containers (diameter $7 \mathrm{~cm}$, height $4 \mathrm{~cm}$ ) in which they were maintained individually until emergence. Ten offspring from each female (family) were reared, resulting in a total of 1470 individuals at the start of this experiment. Throughout the experiment, the individual larvae were fed daily with an average of 350 (SE: 26.8, $N=10$ ) laboratoryreared Artemia salina nauplii.

The temperature simulation was stopped on 25 July for high-latitude $\left(20.2{ }^{\circ} \mathrm{C}\right), 15$ August for central-latitude $\left(21^{\circ} \mathrm{C}\right)$ and 12 September for low-latitude $\left(22^{\circ} \mathrm{C}\right)$ larvae because on these dates in natural conditions temperatures begin to fall. At this date, some individuals that had not emerged remained in the incubators. Therefore, these temperatures were maintained until the end of the experiment, that is until emergence of the last high-latitude individual (7 February 2014), which corresponded to 17 October, 26 September and 3 September for high-, central- and low-latitude populations, respectively. Photoperiods in all incubators followed natural changes until the end of the experiment. For graphical visualization of thermo-photoperiods, see Fig. S1.

\section{Experiment 2: common garden}

The purpose of this experiment was to estimate the genetic variance and covariance structure of the three traits for populations at the three latitudinal gradient positions under conditions that simulated a novel environment with regard to temperature and light regime. We then used these data to compare whether the genetic variance-covariance matrix differed between the simulated and common garden conditions. Therefore, we reared larvae from hatching until emergence in common garden conditions. Specifically, we grew high-, central- and low-latitude larvae under a constant thermo-photoperiod corresponding to the average thermo-photoperiod of the three sampled latitudes over the growth period. Because they do not experience constant light or temperature regime during ontogeny, this environment was novel to all populations. However, the environment differed in time constraints, because a very late season was simulated for the southern populations and a late season for the central and a somewhat late season for the northern ones. With regard to temperature, the environment simulated the week with highest average daily temperature experienced for the central population, and therefore, the northern and southern populations experienced a somewhat higher and lower temperature, respectively, than the average daily maximum over the season. We randomly picked six eggs from eight families (four from 
each population) from each latitude position at the end of the simulated winter period. Offspring from these eight females were also used in the simulated experiments described above. This gave a total of 144 larvae at the start of common garden experiment. We placed these eggs in a fourth incubator with a temperature of $21.9^{\circ} \mathrm{C}$ and photoperiod 19:25-04:35 L-D. We chose this temperature because all populations used in this experiment experienced it for at least several hours a day during midsummer (SS unpublished data). The photoperiod chosen matched the longest day length during the growth season at a mid-latitude location along the transect of our study latitudes $\left(55^{\circ} \mathrm{N}\right)$.

\section{Response variables}

Lestes sponsa eggs overwinter in a fixed embryonic stage (Jödicke, 1996), and we measured individual egg development time as number of days between the date of initiation of spring and the date of hatching. Larval development time was estimated as number of days between the date of hatching (day 1) and the date of emergence. Larval growth rate was estimated as final instar larva head width/larval development time (days) between hatching and emergence. We used larval head width instead of adult head width, because a high proportion of larvae failed to emerge successfully, and head width measurements are unreliable on insects that do not successfully emerge. We also used larval head width because the trait strongly correlates with other size measurements in odonates (Corbet, 1999), including adult mass (Mikolajewski et al., 2004). We did not use size at emergence as a response variable in our analyses because this trait is a combined component of development time and growth rate. Admittedly also growth rate is determined by size, but we chose to use only one of these two covarying traits. In addition, past studies on insects have found that adult size is explained by variation in growth rate rather that development time (Simons et al., 1998).

\section{Statistical methods}

Estimation of genetic correlations was performed using a set of general linear mixed models, specifying proper (co)variance structures. Because the model with all considered phenotypic traits was not stable and exhibited convergence problems, we analysed the traits in pairs (each model estimated parameters for a specific pair of traits). Analyses were based on full-sib genealogical relationships (i.e. omitting the dam random term in mixed models). This approach has been used successful in past studies to partition variance in similar genetic analyses (Shama et al., 2011; Sniegula et al., 2014). Moreover, the extent of maternal effects in our study species (major potential inflator of broad-sense genetic parameters) is negligible (Sniegula et al., 2016a).
Damselflies are often sexually dimorphic in life-history traits (De Block \& Stoks, 2003; Johansson et al., 2005; Sniegula et al., 2016b). Therefore, we included sex in the analyses (see Tables S2 and S3), and thus, genetic correlations/variance estimates presented in the results account for possible differences between sexes (as well as latitudinal differences in trait means). We do however not discuss sex-specific results in our discussion, as we had too few replicates of each sex for a comprehensive interpretation of sex effects.

The full-sib approach estimates the genetic (co)variance in each trait as twice the (co)variance associated with the sire (or genetic family) term. Initially, we included population ID within regions as an additional random term in the analyses. This inclusion did not influence final results, and therefore, we merged populations within regions in all models to increase the power of genetic (Co)variance analyses. All models were analysed in ASReml-R v. 3.0 software.

\section{Simulated conditions}

Our primary goal was to estimate genetic covariances between analysed traits and the differences in these covariances between the three latitudes. Thus, we fitted a series of models that varied in the degree of (co)variance matrix structuring (Lynch \& Walsh, 1998), Table 1. In all cases, the matrices might exhibit two levels of heterogeneity: (co)variances of traits differing between regions (henceforth referred to as separate/one estimate(s) of $\mathrm{G}$ across regions) and trait variances differing between traits within regions (henceforth referred to as heterogeneous $G$ variances). Table 1 lists in detail all fitted models. In all models (except for numbers 5 and 6), residual (co)variances were fully unconstrained (allowing for nonzero residual

Table 1 Structure of all fitted models, described from the point of view of genetic and residual components: Regional G, whether G-matrices are specific for different regions; Het. G, whether G-matrices within regions allow for different trait genetic variances; $\operatorname{cov}_{\mathrm{G}}$, whether genetic covariance between traits is estimated; Het. R, whether residual matrices within regions allow for different trait residual variances; $\operatorname{cov}_{\mathrm{R}}$, whether residual covariance between traits is estimated.

\begin{tabular}{llllll}
\hline Model id & Regional G & Het. G & Cov $_{G}$ & Het. R & cov $_{R}$ \\
\hline 1 & Yes & Yes & $\neq 0$ & Yes & $\neq 0$ \\
2 & Yes & Yes & $=0$ & Yes & $\neq 0$ \\
3 & No & Yes & $\neq 0$ & Yes & $\neq 0$ \\
4 & No & Yes & $=0$ & Yes & $\neq 0$ \\
5 & Yes & Yes & $=0$ & Yes & $=0$ \\
6 & Yes & Yes & $=0$ & No & $=0$ \\
7 & Not entirely* & Yes & $\neq 0$ & Yes & $\neq 0$ \\
\hline
\end{tabular}

*Regional G-matrices but genetic covariances between traits fixed to be equal between regions. 
covariances) and allowed to differ between regions. To avoid biases resulting from inadequately specified residual (co)variances, we always used heterogeneous residual variances (i.e. allowed for different residual variances for different traits in different regions), with estimated residual covariances (which might have contributed to the above-mentioned lack of convergence in some of the preliminary models; however, with different traits, the model should always allow for differing residual (co)variances to avoid biased estimates of genetic parameters). The models were compared in pairs to test relevant hypotheses using the likelihood ratio test. The likelihood ratio tests used twice the difference in likelihood of respective models as the test statistic, assumed to follow a chi-squared distribution when d.f. equal to the number of additional (co)variance parameters estimated by the more complex model in each pair. The rationale behind each of the pairwise comparison is given in the right hand of Table 2 .

\section{Simulated vs. common garden conditions}

Analysis of genetic correlations in individuals reared under common garden conditions was carried out to compare the G-matrix structure with that in the simulated conditions. We analysed the measured traits in similar pairs of characters, estimating separate (co)variance matrices for individuals reared in common garden and simulated native conditions. This analysis involved five types of models, Table 4: (la) genetic (G) and residual $(R)$ variances different in common garden $(\mathrm{cg})$ and simulated condition (sim) groups, genetic and residual covariances between traits $\left(r_{\mathrm{g}}\right.$ and $\left.r_{\mathrm{e}}\right)$ equal to zero; (2a) G and $\mathrm{R}$ covariances uniform across $\mathrm{cg}$ and sim groups, $r_{\mathrm{g}}=0$; (3a) only $\mathrm{R}$ covariances different between cg and sim groups, $r_{\mathrm{g}}=0, r_{\mathrm{e}}=0$; (4a) G and R variances different in $\mathrm{cg}$ and sim groups, $r_{\mathrm{g}} \neq 0, r_{\mathrm{e}}=0$; (5a) $\mathrm{G}$ and $\mathrm{R}$ variances different in $\mathrm{cg}$ and sim groups, $r_{\mathrm{g}} \neq 0, \quad r_{\mathrm{e}} \neq 0$; and (6a) identical to $5 \mathrm{a}$, but genetic covariances forced to be equal between the simulated and common garden conditions. The models were chosen because they represent progressively more complex hypotheses, from completely treatment-specific matrices to complete uniformity across the $\mathrm{cg} / \mathrm{sim}$ groups. Models were compared with likelihood ratio tests in a similar manner. In these analyses, we did not consider regions for two reasons. First, the analysis on the simulated data set (see previous paragraph and Results) showed no strong evidence of differences in the Gmatrix among regions. Second, the common garden data set had too few replicates per region to accommodate this additional level of structuring in the G-matrices. Additionally, the comparison between larval development time and growth rate was not analysed, because we had too few replicates to run the model. However, we present graphical results for regions based on phenotypic correlations. It is important to note that comparisons here are made using two groups with differing sample sizes (147 families in total for simulated conditions, 24 families for common garden experiment). However, as in both cases they were sampled from the identical populations, our results will

Table 2 Pairwise model comparisons. For model identifiers, please see Methods.

\begin{tabular}{|c|c|c|c|c|}
\hline Models & Log-likelihood ratio & d.f. & $P$ & Comparison interpretation \\
\hline \multicolumn{5}{|c|}{ Egg development time and growth rate } \\
\hline 5 vs. 6 & 601.09 & 4 & $<0.001$ & Residual variances different between regions \\
\hline 2 vs. 5 & 66.69 & 3 & $<0.001$ & Residual covariances different from zero \\
\hline 2 vs. 4 & 33.51 & 4 & $<0.001$ & Genetic variances different between regions \\
\hline 3 vs. 4 & 12.35 & 1 & $<0.001$ & Genetic covariances between traits different from zero (ignoring region differences in variances) \\
\hline 1 vs. 3 & 42.05 & 6 & $<0.001$ & Region-specific (co)variance matrices rather than a single (co)variance matrix for traits \\
\hline 1 vs. 2 & 20.89 & 3 & $<0.001$ & Assuming region-specific $\mathrm{G}$, genetic correlations are nonzero \\
\hline 7 vs. 1 & 0.03 & 2 & 0.967 & Constraining genetic covariances to be identical; yields an equally good fit \\
\hline \multicolumn{5}{|c|}{ Egg development time and larval development time } \\
\hline 5 vs. 6 & 603.55 & 4 & $<0.001$ & Residual variances different between regions \\
\hline 2 vs. 5 & 69.45 & 3 & $<0.001$ & Residual covariance different from zero \\
\hline 2 vs. 4 & 32.58 & 4 & $<0.001$ & Genetic variances different between regions \\
\hline 3 vs. 4 & 13.39 & 1 & $<0.001$ & Genetic covariance between traits different from zero (ignoring region differences in variances) \\
\hline 1 vs. 3 & 39.93 & 6 & $<0.001$ & Region-specific (co)variance matrices rather than a single (co)variance matrix for traits \\
\hline 7 vs. 1 & 0.34 & 2 & 0.712 & Constraining genetic covariances to be identical; yields an equally good fit \\
\hline \multicolumn{5}{|c|}{ Larval development time and growth rate } \\
\hline 5 vs. 6 & 56.91 & 4 & $<0.001$ & Residual variances different between regions \\
\hline 2 vs. 5 & 933.40 & 3 & $<0.001$ & Residual covariance different from zero \\
\hline 2 vs. 4 & 0.32 & 4 & 0.960 & Genetic variances not different between regions \\
\hline 3 vs. 4 & 11.32 & 1 & $<0.001$ & Genetic covariance between traits different from zero (ignoring region differences in variances) \\
\hline 1 vs. 3 & 1.70 & 6 & 0.760 & Region-specific (co)variance matrices rather than a single (co)variance matrix for traits \\
\hline
\end{tabular}


not be biased (in both cases random families represent an unbiased sample from all possible family effects present in the population). Moreover, as we were not interested in cross-experiment genetic correlations (i.e. correlations between the common garden and simulated conditions), we were not restricted to using only individuals belonging to the same families.

\section{Results}

\section{Simulated native conditions: question 1}

Patterns of genetic correlations differed substantially between different pairs of traits (Tables 2 and 3). However, no evidence for region-specific patterns was found for any genetic correlation between traits (Table 2: model 7 vs. 1). Nevertheless, genetic variance differed among regions for all traits and was considerably lower in the north (Tables 2 and 3 ).

In two pairs of traits, egg development time-growth rate and egg development time-larval development time, initial evidence indicated region-specific (co)variance matrices (Table 2: 1 vs. 3). However, this pattern was driven mostly by regional differences in genetic variances in specific traits (Table 3). More specifically, for egg development time-growth rate and egg development time-larval development time trait pairs, models with genetic covariances not fixed at zero but constrained to be uniform performed equally well compared with fully unconstrained models (Table 2: l vs. 7), providing no support for region-specific genetic correlations between traits. For larval development rate vs. larval growth rate, the G-matrices were apparently homogenous between regions (models 1 vs. 3 comparison), and therefore, we did not run the model comparisons 7 vs. 1 and 2 vs. 1 .

Overall patterns of genetic correlations supported strong positive genetic correlations between egg development time and growth rate (ranging from 0.88 to 0.94 between regions) and strong negative genetic correlations for egg development time-larval development time (range -0.90 to -0.99 ) and larval development time-growth rate (range -0.94 to -0.99 ; Table 3). Phenotypic correlations are shown graphically in Fig. 2.

In two cases (egg development time-growth rate and egg development time-larval development time), residual covariances showed clear differences between regions (Table 2: 1 vs. 2 and Table 3 ). This result suggested that nongenetic environmental factors and effects not accounted for in our models were mostly responsible for between-region differences in trait correlations observed in our data at the phenotypic level.

\section{Common garden vs. simulated conditions: question 2}

The estimates of $\mathbf{G}$-matrices in the common garden experiment were different from those estimated under simulated natural temperature and photoperiod conditions. Two of the analysed pairs in the common garden conditions showed weaker genetic correlations: egg development time-growth rate; $r_{\mathrm{g}, \mathrm{cg}}=0.25 \pm 0.39$ and $r_{\mathrm{g}, \mathrm{sim}}=0.75 \pm 0.025$ for common garden $(\mathrm{cg})$ and simulated (sim) conditions, respectively, and egg

Table 3 Genetic and residual correlations in pairs of traits at the three analysed latitudes.

\begin{tabular}{|c|c|c|c|c|c|c|}
\hline Traits & Region: Southern & & Region: Central & & Region: Northern & \\
\hline \multirow[t]{2}{*}{ Egg dev. time ${ }^{*}$ vs. growth rate $\dagger$} & $\begin{array}{l}0.21 \pm 0.07 \\
0.82 \pm 0.05\end{array}$ & $0.88 \pm 0.13$ & $\begin{array}{l}0.08 \pm 0.03 \\
0.26 \pm 0.02\end{array}$ & $0.90 \pm 0.08$ & $\begin{array}{r}0.002 \pm 0.001 \\
0.02 \pm 0.001\end{array}$ & $0.94 \pm 0.19$ \\
\hline & $0.50 \pm 0.05$ & $\begin{array}{l}0.14 \pm 0.07 \\
1.49 \pm 0.14\end{array}$ & $0.57 \pm 0.04$ & $\begin{array}{l}0.13 \pm 0.06 \\
0.78 \pm 0.07\end{array}$ & $0.03 \pm 0.07$ & $\begin{array}{l}0.09 \pm 0.04 \\
0.51 \pm 0.06\end{array}$ \\
\hline Egg dev. time* vs. Larval dev. time $\dagger$ & $-0.45 \pm 0.05$ & $\begin{array}{l}0.10 \pm 0.05 \\
1.35 \pm 0.12\end{array}$ & $-0.60 \pm 0.04$ & $\begin{array}{l}0.11 \pm 0.05 \\
0.73 \pm 0.06\end{array}$ & $-0.07 \pm 0.06$ & $\begin{array}{l}0.04 \pm 0.02 \\
0.38 \pm 0.04\end{array}$ \\
\hline Larval dev. time* vs. growth rate $\dagger$ & $\begin{array}{l}0.07 \pm 0.05 \\
1.20 \pm 0.11\end{array}$ & $-0.94 \pm 0.05$ & $\begin{array}{l}0.09 \pm 0.04 \\
0.66 \pm 0.05\end{array}$ & $-0.96 \pm 0.02$ & $\begin{array}{l}0.04 \pm 0.02 \\
0.38 \pm 0.04\end{array}$ & $-0.99 \ddagger$ \\
\hline
\end{tabular}

Each $2 \times 2$ submatrix (i.e. combination of region and traits' pair) provides estimates of relevant variances (diagonal elements, top valuegenetic variance; bottom value-residual variance) and correlations (upper off-diagonal-genetic variance; lower off-diagonal-residual variance). Standard errors were estimated using the delta method.

*Traits represented in rows of covariance matrices.

$\dagger$ Traits represented in columns of covariance matrices.

$\ddagger$ Correlations constrained at the space boundary of the parameters (i.e. close to \pm 1 ). 

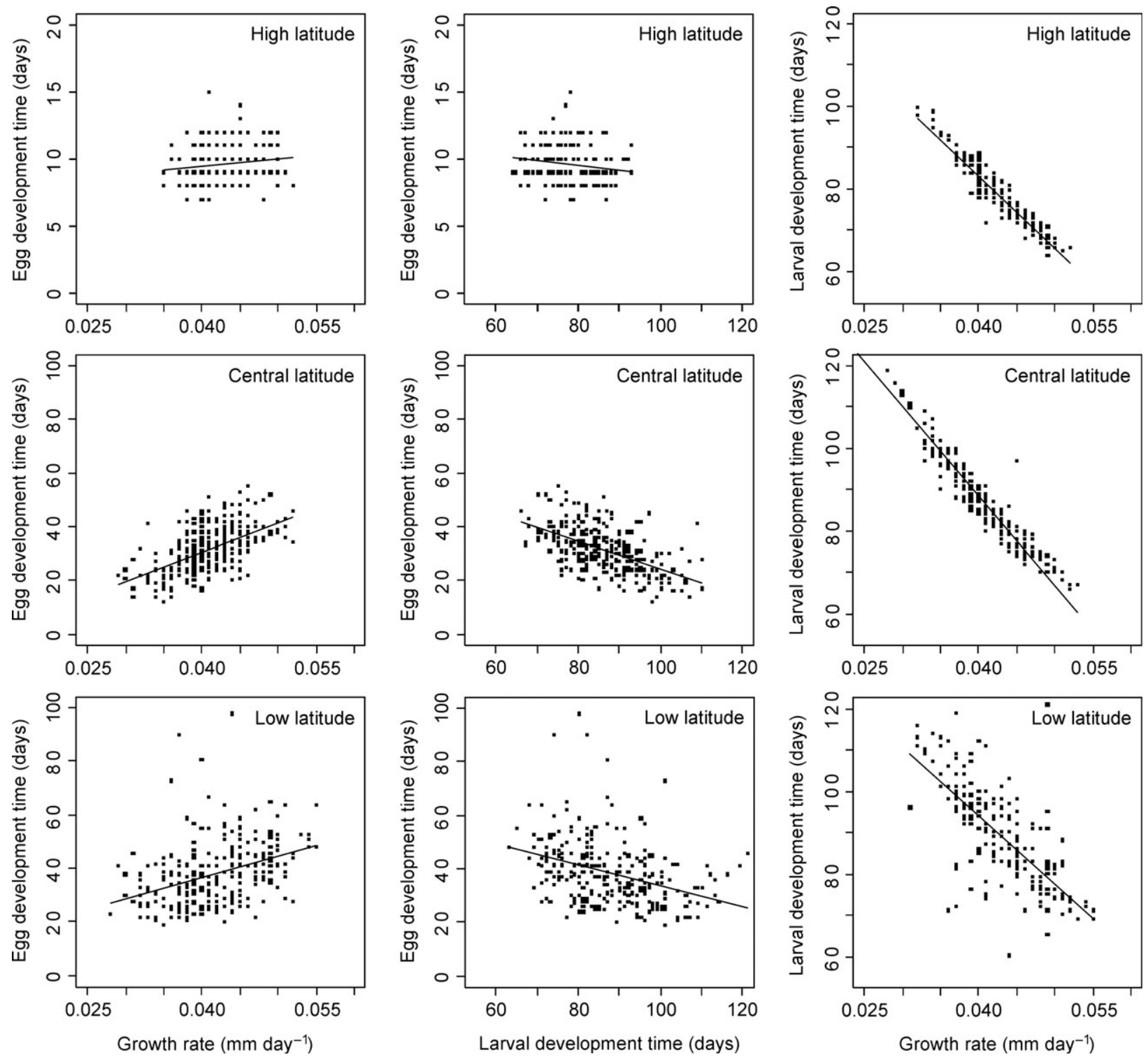

Fig. 2 Phenotypic correlations between larval growth rate $\left(\mathrm{mm}_{\text {day }}{ }^{-1}\right)$-egg development time (days), larval development time (days)-egg development time and larval growth rate-larval development time across high-, central- and low-latitude populations of Lestes sponsa grown in simulated conditions. Residual least square lines are shown (for residual correlation coefficients see Table 3). Note that for egg development time in high-latitude populations, the $y$-axis values differ from those of other latitude populations for better visualization. Correlation coefficients based on raw data with $95 \% \mathrm{CI}$ in brackets and $P$-values: growth rate-egg development time, high-latitude, $r=0.16(0.02 ; 0.29), P=0.022$, central latitude, $r=0.58(0.50 ; 0.65), P<0.001$, low latitude, $r=0.42(0.32 ; 0.51), P<0.001 ;$ larval development time-egg development time, high latitude, $r=-0.17(-0.30 ;-0.03), P=0.015$, central latitude, $r=-0.57(-0.64 ;-0.50)$, $P<0.001$, low latitude, $r=-0.39(-0.48 ;-0.28), P<0.001$; growth rate-larval development time, high latitude, $r=-0.94(-0.96$; $-0.92), P<0.001$, central latitude, $r=-0.95(-0.96 ;-0.94), P<0.001$, low latitude, $r=-0.94(-0.95 ;-0.93), P<0.001$.

development time vs. larval development time; $r_{\mathrm{g}, \mathrm{cg}}=$ $0.50 \pm 0.27$ and $r_{\mathrm{g} \text {, sim }}=-0.91 \pm 0.26$ for common garden and simulated conditions, respectively. The Gmatrix between these two trait pairs differed significantly between the two treatments, as indicated by the model comparisons la vs. $2 a$ and la vs. 3a (Table 4). However, the genetic correlations did not differ between the two treatments for either of the two trait pairs (5a vs. 6a; Table 4). Sample size limitations inflating estimates of standard errors likely explained the absence of a significant difference. Nevertheless, the magnitude of effects (i.e. the correlations themselves) in the simulated treatment for this analysis matched those estimated in the larger analysis above (simulated 
Table 4 Model comparisons for data comparing individuals reared in common garden and simulated conditions. In descriptions, 'treatment' refers to two opposing groups: common garden vs. simulated rearing conditions.

\begin{tabular}{|c|c|c|c|c|}
\hline Models & Log-likelihood ratio & d.f. & $P$ & Comparison interpretation \\
\hline \multicolumn{5}{|c|}{ Egg development time and growth rate } \\
\hline $1 \mathrm{a}$ vs. $2 \mathrm{a}$ & 191.73 & 2 & $<0.001$ & Model with both $G$ and $R$ matrices depending on treatment fits data better \\
\hline $1 \mathrm{a}$ vs. $3 a$ & 183.97 & 1 & $<0.001$ & G-matrix depends on treatment after accounting for $\mathrm{R}$ matrix dependence on treatment \\
\hline $4 a$ vs. $1 \mathrm{a}$ & 4.35 & 1 & 0.003 & Residual correlation(s) between traits differ from zero \\
\hline $5 a$ vs. $4 a$ & 2.51 & 1 & 0.024 & Genetic correlation(s) between traits differ from zero \\
\hline $5 a$ vs. $1 a$ & 6.87 & 1 & $<0.001$ & Genetic correlation(s) between traits differ from zero \\
\hline $5 a$ vs. $6 a$ & 0.33 & 1 & 0.460 & Difference between correlations in treatment groups not statistically significant \\
\hline \multicolumn{5}{|c|}{ Egg development time and larval development time } \\
\hline $1 \mathrm{a}$ vs. $2 \mathrm{a}$ & 192.98 & 2 & $<0.001$ & Model with both $\mathrm{G}$ and $\mathrm{R}$ matrices depending on treatment fits data better \\
\hline $1 \mathrm{a}$ vs. $3 a$ & 184.85 & 1 & $<0.001$ & G-matrix depends on treatment after accounting for $\mathrm{R}$ matrix dependence on treatment \\
\hline $4 a$ vs. $1 \mathrm{a}$ & 2.59 & 1 & 0.023 & Residual correlation(s) between traits differ from zero \\
\hline $5 a$ vs. $4 a$ & 2.25 & 1 & 0.033 & Genetic correlation(s) between traits differ from zero \\
\hline $5 a$ vs. $1 \mathrm{a}$ & 4.84 & 1 & 0.002 & Genetic correlation(s) between traits differ from zero \\
\hline
\end{tabular}

conditions). These results suggested that a difference occurred in the genetic correlation between the two treatments (simulated vs. constant conditions), although further confirmation is required in studies with more statistical power than ours. Phenotypic correlations from the common garden experiment are shown graphically in Fig. S2.

\section{Discussion}

We found strong genetic and phenotypic correlations in the characters shaping growth and development across and within populations. Due to the fact that the growth season is short at northern latitudes, northern populations of this species are more time-constrained than southern ones. This difference in time constrains is accentuated by species-specific life histories, that is an obligatory univoltine damselfly overwintering in the egg stage that must complete whole larval development, emerge and breed within a season (Corbet, 1999; Sniegula et al., 2016c). The evidence for regionspecific phenotypic variance-covariance matrices along the latitudinal gradient was weak, and the genetic (co)variance structures did not differ between the regional latitudes (Table 3). By contrast, genetic variance for single traits differed between latitudes, with variance lower in the north. Thus, the answer to question one is that genetic variance is lower in the north but that the genetic covariance does not differ between latitudes. We emphasize that this pattern was found under native conditions which is the relevant measure at these latitudes for describing the current variancecovariance matrix. Notably, the direction and shape of G-matrices changed when populations were grown in common garden conditions compared with the simulated natural photoperiod and temperature dynamics (Table 4). Hence, the answer to question 2 is that the novel environment changed the estimated genetic architecture of traits to some extent. This result underlines the importance of environmentally induced changes in multivariate genetic structure. Admittedly, a full factorial design would have provided more information on the variance-covariance along the studied gradient.

We found strong positive genetic correlation between growth rate of larvae and egg development time. We suggest that a positive correlation is caused by selection on compensatory growth. Individuals that require a longer time for egg development and therefore hatch at later dates are selected for faster growth to reach threshold larval size and emerge before the end of the season. Additionally, the most time-constrained highlatitude females produce larger offspring (eggs and hatchlings) than less time-stressed central and southern females, despite having the smallest adult size (Sniegula et al., 2016b). These time-constrained northern populations also have the highest growth rate (Sniegula et al., 2016a). Large offspring might have several advantages in time-constrained populations (Eckerström-Liedholm et al., 2017); for example, large size at hatching allows an increase in threshold size for prey capture, resulting in increased food intake and growth rate (Hirvonen $\mathcal{E}$ Ranta, 1996; Karl \& Fischer, 2008).

A negative genetic relationship was observed between larval development time and egg development time, which suggests a genetic trade-off between these two traits. As L. sponsa most likely is very time-constrained because of overwintering eggs, we suggest that a long egg development time must be compensated by a short larval period, because adults must emerge before the onset of winter. Additionally, we found a negative genetic correlation between larval development time and growth rate, which is a common pattern in many organisms (reviewed in Dmitriew, 2011), 
because a high growth rate results in decreased time to emergence into the adult stage.

Our results showed weak divergence in genetic correlations between traits among the studied regions. For all three pairwise correlations, the whole G-matrix differed significantly between regions, but we found no significant difference between regions in any of the three pairwise comparisons for the genetic correlations. This result suggests that the differences observed in the G-matrix were caused by differences in genetic variance between regions. Using the same data set, we showed previously that the genetic variance for these traits is significantly lower in the northern regions (Sniegula et al., 2016a), and the same pattern was found in the current analysis. These northern populations consist of several hundreds of individuals and are surrounded by other populations. Hence, even though the role of drift cannot be excluded, it seems likely that our results are due to stronger selection in the north. Estimates on effective population size (Ne) would help to clarify this. One would therefore also expect a stronger correlation between pairwise traits in northern regions compared with southern regions. The absence of a stronger correlation in the north in our study could be because these life-history traits are tightly connected by important trade-offs similarly in all regions and would work in the same direction in this strongly time-constrained species. Hence, it would be very costly to break them apart. We suggest that this would probably not apply to ectotherms that are not obligate univoltine, that is that can increase their generation number or voltinism when growth season becomes longer, that is, towards lower latitudes, or vice versa. In this case, organisms often show more complex responses to environmental variables at the phenotypic level (variation in number of generations per season in relation to environmental variables, e.g. Kivelä et al., 2011; Sniegula et al., 2012b).

We acknowledge that the results discussed above could be at least partly due to nonadditive (dominance, epistasis and/or maternal) effects: life-history traits measured in this experiment can harbour considerable nonadditive genetic effects (Roff, 1997) that could not be partitioned in full-sibling analyses. However, our previous analyses based on half-sibling experimental design indicated that maternal effects influenced up to $12 \%$ of genetic variance in measured traits across studied populations (Sniegula et al., 2016a). Although a $12 \%$ effect is not negligible, maternal effects should not be the major drivers of potential evolutionary changes in the study system.

In general, we found that genetic and phenotypic correlations were similar in sign and strength. Based on this result, the environmental effects had the same effect as those of the genetic effects in simulated native conditions. Results from our previous experiment where we grew populations of L. sponsa originating from different latitudes in different photoperiods (a key environmental factor that shapes life history in temperate odonates) indicated the presence of a strong phenotypic plasticity in life-history traits (Sniegula et al., 2014). In that experiment, a long day length increased larval growth and development rate compared to a short day length in all studied populations, indicating strong and adaptive environmental effects (Sniegula et al., 2014). However, there was low genetic variance and hence weak evolutionary potential of reaction norms in development time and growth rate (Sniegula et al., 2014) - similarly to our current results for genetic variance in individual traits measured in individuals grown in simulated native conditions.

The strong divergence in genetic variances of the Gmatrix structures (but with no divergence in genetic correlations) between regions is consistent with many other studies that have compared the G-matrix among populations within a species (e.g. Brodie, 1993; Podolsky et al., 1997; Ashman, 2003; Cano et al., 2004; Arnold et al., 2008; Teplitsky et al., 201 1; Delahaie et al., 2017).

In contrast to our study, Paccard et al. (2016) found that genetic correlations were weaker at the range margins (south and north) of Arabidopsis lyrata, which they interpreted as a consequence of stronger genetic drift at range margins. However, as we argue above, our populations in the northern region consisted of hundreds of individuals, in addition to other nearby populations; therefore, drift might be of less importance in our study. Additionally, and perhaps more importantly, Paccard et al. (2016) estimates of genetic variance and covariance were from a common garden environment and thus not at the natural condition the plants experience. Therefore, direct comparisons with our results are difficult as we used natural environmental condition. In addition, a comparison with our common garden results is unrealistic as the two common garden experiments (theirs and ours) differ very much in their environmental conditions.

Our results also highlight the importance of studying potential changes of life-history traits using a variancecovariance approach. If traits had been studied individually, we would have found that southern populations had a higher potential to respond to selection, because these populations showed a higher variance in the traits studied, as also found in Sniegula et al. (2016a). Notably, the genetic covariances did not differ between regions, and therefore, the responses of the populations across the latitudinal gradient would not be confounded by genetic covariance differences between latitudes for the traits studied. This is not always the case, and the difficulty of predicting evolutionary responses of lifehistory traits using isolated traits are noted repeatedly (Pigliucci \& Preston, 2004; Brookfield, 2016). For example, Paccard et al. (2016) concluded that variation in 
isolated traits of Arabidopsis lyrata would be a poor predictor of potential selection in their study because of the structure of the genetic correlations between the traits studied.

Although genetic variation for life-history traits was low and genetic correlations between the traits were strong in the northern region, we cannot predict that these populations would respond faster or slower to environmental change than those at more southern regions in which variances were higher. The reason is that environmental change might release heritable variation in a new environment (cryptic genetic variation). Such variation likely facilitates adaptation in a new environment because phenotypic buffering mechanisms are disrupted (Paaby $\&$ Rockman, 2014). In fact, our common garden experiment suggested this, because genetic correlation became weaker in the common garden experiment. However, predictions must be based on realistic environmental change. We used a common garden approach to simply explore how and whether the G-matrix changed; therefore, in our case, simulation of future predicted temperature change and effects on the G-matrix would be of interest. Nevertheless, the conditions chosen for the common garden experiment could be interpreted as a strong seasonal time constraints for newly hatched individuals, and the most so for southern latitude populations and the least so for northern latitude populations. The northern population would need to experience much longer day length for strong time constraint (Sniegula et al., 2016a,c).

Several empirical studies show that the environment has a strong effect on the G-matrix (Johansson et al., 2012; Sikkink et al., 2015; Green et al., 2016), and in a review, Wood \& Brodie (2015) found that between environmental effects were equal to or stronger than between-population effects. Our study supports the finding of their review, because we found large differences within populations in the G-matrix depending on whether populations were raised in a common garden experiment with constant temperature and photoperiod or in an environment that simulated natural changes in photoperiod and temperature. The main cause of the difference in the G-matrix in our study was that the genetic variance differed along the gradient. Nevertheless, our results highlight the importance of the environment in the understanding of the $\mathbf{G}$ matrix. We emphasize that is important to design the experiment carefully when the goal is to understand the structure of the $\mathbf{G}$-matrix under natural conditions and to determine the effects of environmental changes on the matrix structure (Conner et al., 2003). A common approach in G-matrix studies is to move an organism to a common garden environment and then use the results from such an experiment to make predictions on the effects on environmental change. However, for a qualitative prediction on changes between natural environments, we suggest to conduct experiments where organisms are grown in different environmental conditions, preferably in conditions that mimic native ones as well as those that are predicted by climate models. This is because the changes in Gmatrix observed are very environment-specific as shown in our study and in others (Wood $\&$ Brodie, 2015; Brookfield, 2016). With a common garden approach to describe the genetic correlation differences between regions, a very different pattern would have emerged in our study. With those results, the interpretation would have been that the traits were less genetically correlated than they actually were at their origin of sampling and environment. Hence, one should be aware of which question is undertaken by each experimental set-up.

\section{Acknowledgments}

We thank The Nature Reserve Marais du Vigueirat, France, for permission to sample and Philippe Lambret and Viktor Nilsson-Örtman for information about French populations. We thank Martin Lind for comments on the first draft of the manuscript. Szymon Sniegula, Maria J. Golab and Szymon M. Drobniak are funded by National Science Centre (SS, grant UMO2012/05/N/NZ8/00981 and scholarships Etiuda 2014/ 12/T/NZ8/00522; MJG, scholarship Etiuda 2015/16/T/ NZ8/00392; SMD, grant UMO-2015/18/E/NZ8/00505). Szymon Sniegula and Maria J. Golab are partially funded by the Institute of Nature Conservation Polish Academy of Sciences. Frank Johansson is funded by the Swedish Research Council.

\section{Competing interests}

The authors declare no competing interests.

\section{References}

Agrawal, A.F. \& Stinchcombe, J.R. 2009. How much do genetic covariances alter the rate of adaptation? Proc. R. Soc. Lond. B Biol. Sci. 276: 1183-1191.

Arnold, S.J., Bürger, R., Hohenlohe, P.A., Ajie, B.C. \& Jones, A.G. 2008. Understanding the evolution and stability of the G-matrix. Evolution 62: 2451-2461.

Ashman, T. 2003. Constraints on the evolution of males and sexual dimorphism: field estimates of genetic architecture of reproductive traits in three populations of gynodioecious Fragaria virginiana. Evolution 57: 2012-2025.

Bailey, L.D. \& van de Pol, M. 2016. Tackling extremes: challenges for ecological and evolutionary research on extreme climatic events. J. Anim. Ecol. 85: 85-96.

Blows, M. \& Hoffmann, A. 2005. A reassessment of genetic limits to evolutionary change. Ecology 86: 1371-1384.

Boudot, J.-P. \& Kalkman, V. 2016. Atlas of the European Dragonflies and Damselflies. KNNV Publishing, Zeist, The Netherlands.

Brodie, E. 1993. Homogeneity of the genetic variance-covariance matrix for antipredator traits in 2 natural-populations 
of the garter snake Thamnophis ordinoides. Evolution 47: 844854.

Brookfield, J.F.Y. 2016. Why are estimates of the strength and direction of natural selection from wild populations not congruent with observed rates of phenotypic change? BioEssays 38: 927-934.

Bybee, S., Córdoba-Aguilar, A., Duryea, M.C., Futahashi, R., Hansson, B., Lorenzo-Carballa, M.O. et al. 2016. Odonata (dragonflies and damselflies) as a bridge between ecology and evolutionary genomics. Front. Zool. 13: 46.

Cano, J., Laurila, A., Palo, J. \& Merila, J. 2004. Population differentiation in $\mathrm{G}$ matrix structure due to natural selection in Rana temporaria. Evolution 58: 2013-2020.

Colautti, R.I. \& Barrett, S.C.H. 2011. Population divergence along lines of genetic variance and covariance in the invasive plant Lythrum salicaria in eastern North America. Evolution 65: 2514-2529.

Conner, J.K. \& Hartl, D.L. 2004. A Primer of Ecological Genetics, 1st edn. Sinauer Associates, Sunderland, MA.

Conner, J.K., Franks, R. \& Stewart, C. 2003. Expression of additive genetic variances and covariances for wild radish floral traits: comparison between field and greenhouse environments. Evolution 57: 487-495.

Corbet, P. 1956. The influence of temperature on diapause development in the dragonfly Lestes sponsa (Hansemann) (Odonata: Lestidae). P. Roy. Entomol. Soc. A 31: 45-48.

Corbet, P. 1999. Dragonflies: Behavior and Ecology of Odonata. Harley Books, Colchester, UK.

De Block, M. \& Stoks, R. 2003. Adaptive sex-specific life history plasticity to temperature and photoperiod in a damselfly. J. Evol. Biol. 16: 986-995.

De Block, M. \& Stoks, R. 2005. Fitness effects from egg to reproduction: bridging the life history transition. Ecology 86: 185-197.

Delahaie, B., Charmantier, A., Chantepie, S., Garant, D., Porlier, M. \& Teplitsky, C. 2017. Conserved G-matrices of morphological and life-history traits among continental and island blue tit populations. Heredity 119: 76-87.

Dmitriew, C. 2011. The evolution of growth trajectories: what limits growth rate? Biol. Rev. 86: 97-116.

Doroszuk, A., Wojewodzic, M.W., Gort, G. \& Kammenga, J.E. 2008. Rapid divergence of genetic variance-covariance matrix within a natural population. Am. Nat. 171: 291-304.

Eckerström-Liedholm, S., Sowersby, W., Gonzalez-Voyer, A. \& Rogell, B. 2017. Time-limited environments affect the evolution of egg-body size allometry. Evolution 71: 1900-1910.

Etterson, J.R. \& Shaw, R.G. 2001. Constraint to adaptive evolution in response to global warming. Science 294: 151-154.

Geenen, S., Jordaens, K., De Block, M., Stoks, R. \& De Bruyn, L. 2000. Genetic differentiation and dispersal among populations of the damselfly Lestes viridis (Odonata). J. N. Am. Benthol. Soc. 19: 321-328.

Gotthard, K. 2001. Growth strategies of ectothermic animals in temperate environments. In: Environment and Animal Development: Genes, Life Histories and Plasticity (D. Atkinson and M. Thorndyke, eds), pp. 287-303. Bios Scientific Publisher Limited, Oxford, UK.

Green, K., Eroukhmanoff, F., Harris, S., Pettersson, L. \& Svensson, E. 2016. Rapid changes in genetic architecture of behavioural syndromes following colonization of a novel environment. J. Evol. Biol. 29: 144-152.
Hirvonen, H. \& Ranta, E. 1996. Prey to predator size ratio influences foraging efficiency of larval Aeshna juncea dragonflies. Oecologia 106: 407-415.

IPCC 2013. Climate Change 2013: The Physical Science Basis. Contribution of Working Group I to the Fifth Assessment Report of the Intergovernmental Panel on Climate Change. IPCC, Cambridge University Press, Cambridge, UK.

Jödicke, R. 1996. Die Binsenjungfern und Winterlibellen Europas: Die Libellen Europas Bd. 3, Lestidae. VerlagsKG Wolf, Magdeburg.

Johansson, F., Crowley, P. \& Brodin, T. 2005. Sexual size dimorphism and sex ratios in dragonflies (Odonata). Biol. J. Lin. Soc. 86: 507-513.

Johansson, F., Lind, M., Ingvarsson, P. \& Bokma, F. 2012. Evolution of the G-matrix in life history traits in the common frog during a recent colonisation of an island system. Evol. Ecol. 26: 863-878.

Karl, I. \& Fischer, K. 2008. Why get big in the cold? Towards a solution to a life-history puzzle. Oecologia 155: 215-225.

Kause, A., Saloniemi, I., Morin, J.-P., Haukioja, E., Hanhimäki, S. \& Ruohomäki, K. 2001. Seasonally varying diet quality and the quantitative genetics of development time and body size in birch feeding insects. Evolution 55: 1992-2001.

Kivelä, S.M., Välimäki, P., Carrasco, D., Mäenpää, M.I. \& Oksanen, J. 2011. Latitudinal insect body size clines revisited: a critical evaluation of the saw-tooth model. J. Anim. Ecol. 80: 1184-1195.

Lake Model Flake 2009. Flake. http://www.cosmo-model.org/ content/model/modules/flake/ [Accessed: 2-5-2017].

Lynch, M. \& Walsh, B. 1998. Genetics and Analysis of Quantitative Traits. Sinauer Associates Inc., Sunderland, MA.

McGuigan, K. \& Sgro, C. 2009. Evolutionary consequences of cryptic genetic variation. Trends Ecol. Evol. 24: 305-311.

Mikolajewski, D., Johansson, F. \& Brodin, T. 2004. Conditiondependent behaviour among damselfly populations. Can. J. Zool. 82: 653-659.

O'Connor, C.M., Norris, D.R., Crossin, G.T. \& Cooke, S.J. 2014. Biological carryover effects: linking common concepts and mechanisms in ecology and evolution. Ecosphere 5: 28.

Orizaola, G., Dahl, E. \& Laurila, A. 2014. Compensatory growth strategies are affected by the strength of environmental time constraints in anuran larvae. Oecologia 174: 131-137.

Paaby, A.B. \& Rockman, M.V. 2014. Cryptic genetic variation: evolution's hidden substrate. Nat. Rev. Genet. 15: 247-258.

Paccard, A., Van Buskirk, J. \& Willi, Y. 2016. Quantitative genetic architecture at latitudinal range boundaries: reduced variation but higher trait independence. Am. Nat. 187: 667677.

Pigliucci, M. 2005. Evolution of phenotypic plasticity: where are we going now? Trends Ecol. Evol. 20: 481-486.

Pigliucci, M. \& Preston, K. 2004. Phenotypic Integration: Studying the Ecology and Evolution of Complex Phenotypes, 1st edn. Oxford Univ Press, New York, NY, USA.

Podolsky, R., Shaw, R. \& Shaw, F. 1997. Population structure of morphological traits in Clarkia dudleyana. II. Constancy of within-population genetic variance. Evolution 51: 17851796.

Potter, K.A., Davidowitz, G. \& Arthur Woods, H. 2011. Crossstage consequences of egg temperature in the insect Manduca sexta. Funct. Ecol. 25: 548-556. 
Prokop, Z.M. \& Drobniak, S.M. 2016. Genetic variation in male attractiveness: it is time to see the forest for the trees. Evolution 70: 913-921.

Puentes, A., Granath, G. \& Agren, J. 2016. Similarity in G matrix structure among natural populations of Arabidopsis lyrata. Evolution 70: 2370-2386.

Roff, D. 1997. Evolutionary Quantitative Genetics. Softcover reprint of the original 1st ed. 1997 edition. Springer, New York, NY.

Savell, K.R.R., Auerbach, B.M. \& Roseman, C.C. 2016. Constraint, natural selection, and the evolution of human body form. Proc. Natl. Acad. Sci. USA 113: 9492-9497.

Shama, L., Campero-Paz, M., Wegner, K., De Block, M. $\delta$ Stoks, R. 2011. Latitudinal and voltinism compensation shape thermal reaction norms for growth rate. Mol. Ecol. 20: 2929-2941.

Sikkink, K.L., Reynolds, R.M., Cresko, W.A. \& Phillips, P.C. 2015. Environmentally induced changes in correlated responses to selection reveal variable pleiotropy across a complex genetic network. Evolution 69: 1128-1142.

Simons, A.M., Carrière, Y. \& Roff, D.A. 1998. The quantitative genetics of growth in a field cricket. J. Evol. Biol. 11: 721733.

Sniegula, S. \& Johansson, F. 2010. Photoperiod affects compensating developmental rate across latitudes in the damselfly Lestes sponsa. Ecol. Entomol. 35: 149-157.

Sniegula, S., Johansson, F. \& Nilsson-Örtman, V. 2012a. Differentiation in developmental rate across geographic regions: a photoperiod driven latitude compensating mechanism? Oikos 121: 1073-1082.

Sniegula, S., Nilsson-Örtman, V. \& Johansson, F. 2012b. Growth pattern responses to photoperiod across latitudes in a northern damselfly. PLoS One 7: e46024.

Sniegula, S., Drobniak, S.M., Gołąb, M.J. \& Johansson, F. 2014. Photoperiod and variation in life history traits in core and peripheral populations in the damselfly Lestes sponsa. Ecol. Entomol. 39: 137-148.

Sniegula, S., Golab, M.J., Drobniak, S.M. \& Johansson, F. 2016a. Seasonal time constraints reduce genetic variation in life-history traits along a latitudinal gradient. J. Anim. Ecol. 85: 187-198.

Sniegula, S., Golab, M.J. \& Johansson, F. 2016b. A large-scale latitudinal pattern of life-history traits in a strictly univoltine damselfly. Ecol. Entomol. 41: 459-472.

Sniegula, S., Gołąb, M.J. \& Johansson, F. 2016c. Time constraint effects on phenology and life history synchrony in a damselfly along a latitudinal gradient. Oikos 125: 414-423.
Sniegula, S., Janssens, L. \& Stoks, R. 2017. Integrating multiple stressors across life stages and latitudes: combined and delayed effects of an egg heat wave and larval pesticide exposure in a damselfly. Aquat. Toxicol. 186: 113-122.

Stearns, S., de Jong, G. \& Newman, B. 1991. The effects of phenotypic plasticity on genetic correlations. Trends Ecol. Evol. 6: 122-126.

Stoks, R., Geerts, A.N. \& De Meester, L. 2014. Evolutionary and plastic responses of freshwater invertebrates to climate change: realized patterns and future potential. Evol. Appl. 7: $42-55$.

Teplitsky, C., Mouawad, N.G., Balbontin, J., de Lope, F. \& Moller, A.P. 2011. Quantitative genetics of migration syndromes: a study of two barn swallow populations. J. Evol. Biol. 24: 2025-2039.

Wood, C.W. \& Brodie, E.D. 2015. Environmental effects on the structure of the G-matrix. Evolution 69: 2927-2940.

\section{Supporting information}

Additional Supporting Information may be found online in the supporting information tab for this article: Table $\mathbf{S} 1$ Coordinates for sampled populations.

Table S2 Tests of fixed effects included in mixed-models analyzing latitudinal differences in genetic correlations.

Table S3 Tests of fixed effects included in mixed models analyzing differences in genetic correlations between constant and simulated conditions.

Figure S1 Graphs showing (a) temperatures and (b) photoperiods used during the simulated conditions experiment.

Figure S2 Residual correlations between egg development time (days) - larval growth rate $\left(\mathrm{mm}\right.$ day $\left.{ }^{-1}\right)$, egg development time - larval development time (days) and larval development time - larval growth rate across high-, central- and low-latitude populations of Lestes sponsa grown in common-garden conditions.

Data from simulated natural and common garden experiments: Dryad doi: 10.5061/dryad.2nh8c43.

Received 27 October 2017; revised 15 March 2018; accepted 15 March 2018 